\title{
Active vibration control of lightweight floor systems
}

\section{Conference Paper}

Author(s):

Baader, Jakob; Fontana, Mario

Publication date:

2017

Permanent link:

https://doi.org/10.3929/ethz-b-000236140

Rights / license:

Creative Commons Attribution-NonCommercial-NoDerivatives 4.0 International

Originally published in:

Procedia Engineering 199, https://doi.org/10.1016/j.proeng.2017.09.529 


\title{
Active vibration control of lightweight floor systems
}

\author{
J. Baader ${ }^{\mathrm{a}, *}$, M. Fontana ${ }^{\mathrm{a}}$ \\ ${ }^{a}$ Institute of Structural Engineering (IBK), ETH Zürich, Stefano-Franscini-Platz 5, 8093 Zürich, Switzerland
}

\begin{abstract}
Long-span and lightweight floors are often prone to structural vibrations due to their low resonance frequency and inherent damping. They are easily excited by human walking or running and present a serviceability problem for persons working or living in these buildings. Tuned mass dampers can be used to improve the dynamic behaviour of such structures. The drawback of these passive damping systems is that they require high inertial damper masses and that the damping efficiency in transient phase is low. Especially for systems with resonance frequencies that change over time (due to an addition mass or a change of the structure or temperature dependant phenomena) the damping efficiency drops rapidly.

Active controlled tuned mass damper can be used to reduce the inertial mass and improve the damping efficiency for a broad band of frequencies in transient phase. Several constraints, like the stability in closed loop, robustness and performance criteria and the maximum stroke and saturation of the actuator have to be considered when designing a controller for an active vibration control device. Different control strategies are analysed in this work and simulated using a $2^{\text {nd }}$ and a $4^{\text {th }}$ order system in order to analyse advantages and drawbacks.
\end{abstract}

(C) 2017 The Authors. Published by Elsevier Ltd.

Peer-review under responsibility of the organizing committee of EURODYN 2017.

Keywords: Active Vibration Control, Vibration Mitigation, Human induced floor vibration

\section{Introduction}

Light-weight floors in buildings with big span-widths are often easily excited by vertical disturbances. The source of these disturbance forces are for example people walking or jumping, machines fixed on the floor or external disturbances like traffic or else. The resonance frequencies of these floors are usually in the range of 1 to $5 \mathrm{~Hz}$ and they present inherent damping coefficients below $2 \%$. The resulting vertical vibrations can be very disturbing for people working or living in such a building. Owners and users wish to have open spaces with as little columns as possible in a room. Therefore one can often not encounter the vertical vibration problem by reducing the span-width. Today's solution to the dynamic problems of floors are to either increase the dead-weight of the floor in order to decrease the excitability or to increase the bending stiffness of the floor. Both solutions require a higher amount of building material (usually concrete, timber or steel) which leads to higher costs in planning and construction.

A novel method to increase the dynamic properties of floors is to implement an additional vibration mitigation. The TMD (Tuned Mass Damper) is the most common passive damping device. It consist of an auxiliary spring-damper-

\footnotetext{
* Corresponding author. Jakob Baader, Tel.: +41-446-333-450

E-mail address: baader@ibk.baug.ethz.ch
} 

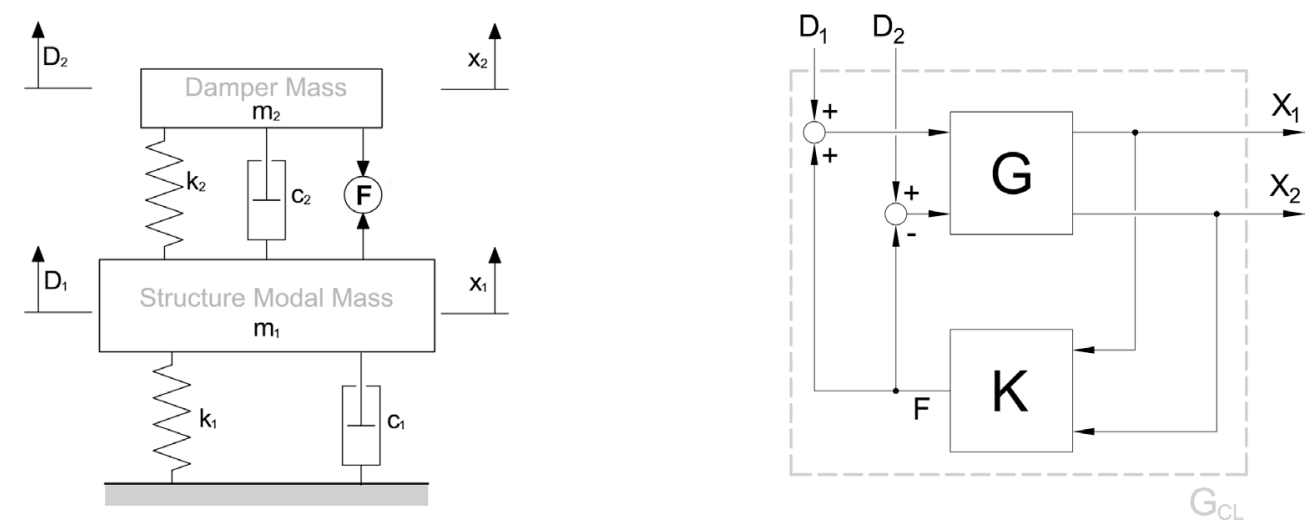

Fig. 1: Schema of an active vibration control device (left figure), complete closed loop control of the plant model $\mathrm{G}$ with the controller $\mathrm{K}$ (right figure). $X$ represents the absolute position of the masses, $D$ is the external disturbance force on the mass and $F$ the actuator force. $G$ and $G_{C L}$ represent the open and closed-loop system respectively.

mass system which is connected to the primary system. If the auxiliary system is correctly tuned, an efficient damping of the primary structure is achieved. TMD's have become standard parts in bridge and skyscraper construction over the past 30 years. Due to a high damper mass (usually between 4 and 10\% of the modal mass of the structure) they are quite robust to slightly changing resonance frequencies of the primary system. For bridges and skyscraper for example several tons [1] of additional dead-weight need to be added to the primary structure. Especially for lightweight constructions this can reduce considerably the load-capacity of the structure. Another drawback of standard TMD's is that the frequency of the primary system needs to be known in the design stage of the TMD. This is often not possible with the required accuracy during the planning of a new building. Therefore the external damping devices are usually applied today only after the completion of the building. Resonance frequencies vary also during the lifetime of a building when heavy equipment or objects are moved or due to maturing of the construction materials. Active vibration control devices can help to encounter these problems. Active vibration mitigation requires at least one actuator which is located between the primary structure and the auxiliary system (cf. figure 1). This helps to control the movement of the auxiliary mass. Thus they can achieve better damping efficiency and require lower damper mass, depending on the applied control law. A drawback is that this leads usually to higher strokes of the auxiliary mass.

The controller is the most important part in active control. It governs the actuator force based on current and past measurements. There are a variety of control strategies which have advantages and drawbacks. Some of these strategies are analysed and compared in this work for the application of vertical floor vibration mitigation. The goal of this work is to investigate the major drawbacks and advantages for each control strategy.

\section{Simulation and Control Schema}

\subsection{Control Schema}

A vibrating structure is simplified to a harmonic oscillator with viscous damping (primary system with $m_{1}, c_{1}, k_{1}$ ). The damper mass $\left(m_{2}\right)$ is placed on the top of the structure, suspended by vertical linear springs $\left(k_{2}\right)$ and damped by a dash-pot $\left(c_{2}\right)$. This results in the well-known structure of a Tuned Mass Damper (TMD). By adding an actuator between the structure and the damper the system becomes an active damping device. According to the tuning of the auxiliary system the active damping device is called "Active Tuned Mass Damper (ATMD) or "Active Mass Driver" (AMD) when no tuning is required. The linear model presented in figure 1 is described by following matrix equation in Laplace domain. It is assumed that all initial values are zero. The coordinates $X_{1}$ and $X_{2}$ represent the absolution position of the main and auxiliary mass, whereas $D_{1}$ and $D_{2}$ are the disturbance forces on the masses.

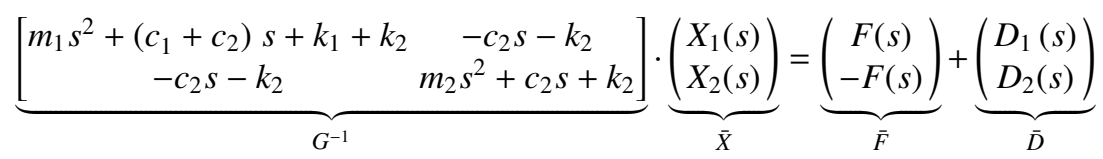


In this work the actuator force $F$ is a linear feedback of the states of the system. The actuator force acts equally on the primary and auxiliary mass with opposite sign. The matrix $\bar{K}$ is the controller. Let therefore be:

$$
\bar{F}=\left[\begin{array}{c}
F(s) \\
-F(s)
\end{array}\right]=\left[\begin{array}{cc}
K_{1}(s) & K_{2}(s) \\
-K_{1}(s) & -K_{2}(s)
\end{array}\right]\left[\begin{array}{c}
X_{1}(s) \\
X_{2}(s)
\end{array}\right]=\bar{K} \cdot \bar{X}
$$

Equation (1) becomes therefore in closed loop $(\bar{G}$ is the plant model and $I$ represents the identity matrix, without showing the dependence on s):

$$
\bar{X}=\left(G^{-1}+\bar{K}\right)^{-1} \cdot \bar{D}=(I+G \bar{K})^{-1} G \cdot \bar{D}
$$

\subsection{Simulation Model}

Two different plant models are used to evaluate the damping efficiency of the chosen control laws. The first plant model corresponds to the damped harmonic oscillator with one resonance frequency and viscous damping described in section 2.1. The second model is a damped oscillator with two resonance frequencies. It models for example the behaviour of a slender beam-type floor structure with an additional orthogonal or torsional mode. The characteristics of the plant model are given in table 1. A damping device with an auxiliary mass of $m_{2}=50 \mathrm{~kg}(\mu=1 \%)$ is applied. An auxiliary resonance frequency of $f_{2}=1.5 \mathrm{~Hz}$ and a damping ratio of $\xi_{2}=3 \%$ are chosen for the non-tuned control laws.

\section{3. (Active) Tuned Mass Damper (ATMD)}

An active-tuned auxiliary system is auto-exited by the vibration of the structure. It works therefore on the same principle as the well-known Tuned Mass Damper. The simplest control law for an ATMD is to adapt the stiffness and damping according to the structure's current resonance frequency (after a change in loading for example). This method is applicable when the dominant frequencies are well separated and the variation is slow. Weber [3] applied it successfully to light-weight steel bridges with two different load scenarios. The drawback of this method is that it requires some time to adapt to a changing resonance frequency. For fast vanishing or broadband disturbances this vibration mitigation device act like a standard TMD. In the simulation only the TMD is therefore considered.

\subsection{Acceleration feedback (Nishimura)}

Nishimura [2] proposed a positive feedback of the acceleration of the structure. This reduces the apparent modal mass of the structure. The control law $f(t)=-m_{d} \ddot{x}_{1}(t)$ is applied and leads to following closed loop behaviour (from equation 3):

$$
X(s)=\left(G^{-1}+\left[\begin{array}{cc}
-m_{d} s^{2} & 0 \\
m_{d} s^{2} & 0
\end{array}\right]\right)^{-1} \cdot D(s)
$$

This leads to higher resonance frequencies of the structure due to the reduced modal mass $\left(m_{1}-m_{d}\right)$. In order to guarantee stability in theory the criteria $m_{d}<m_{1}$ must be fulfilled. Nishimura [2] established a closed form solution for the optimal feedback parameters with two equal response peaks similar to Den Hartog. For the simulations a feedback gain of $m_{d}=960[\mathrm{~kg}]$ is applied. This is equal to $19.2 \%$ of the modal mass of the system.

\subsection{Active Mass Driver (AMD) with velocity feedback}

This control strategy works with a completely detuned auxiliary system. The mutual interaction of the structure and damper is not desired and therefore a very low auxiliary resonance frequency and damping are chosen. The interaction is provided by the actuator only. The closed loop behaviour of the perfectly decoupled damping device is given by the following transfer function for a simple structure with one resonance frequency and velocity feedback control of the structure $f(t)=-c_{d} \dot{x}_{1}(t)$. From (3) follows:

$$
X(s)=\left(\left[\begin{array}{cc}
m_{1} s^{2}+c_{1} s+k_{1} & 0 \\
0 & m_{2} s^{2}
\end{array}\right]+\left[\begin{array}{cc}
c_{d} s & 0 \\
-c_{d} s & 0
\end{array}\right]\right)^{-1} \cdot D(s)
$$


This leads in theory to an additional damping of the primary structure:

$$
X_{1}(s)=\frac{1}{m_{1} s^{2}+\left(c_{1}+c_{d}\right) s+k_{1}} D_{1}(s)
$$

For this case the auxiliary system consists of a perfect second order integrator in closed loop. Low frequency disturbances are therefore strongly amplified. In reality the auxiliary system needs a physical connection to the structure. Usually a low-frequency suspension with coil-springs is chosen. This leads to an additional resonance frequency of the auxiliary system which is strongly amplified in closed loop and can lead to stability issues. The auxiliary resonance must therefore be far from the structures resonance frequency. This control strategy has been investigated by Hudson [4]. For low frequency applications it is not always possible to build a vibration control device with the desired low resonance frequency due to physical limitations. In order to further reduce the resonance frequency of the auxiliary system, a negative stiffness or additional damping can be overlaid to the calculated control force.

For the simulation a velocity feedback gain of $c_{d}=4^{\prime} 500[\mathrm{~kg} / \mathrm{s}]$ is applied. An improvement of the auxiliary resonance is achieved when an additional viscous damping of the auxiliary system of $200 \mathrm{~kg} / \mathrm{s}$ is added.

\subsection{Disturbance estimation}

The disturbance force causing the vibration of the main structure is not directly measurable in the configuration of floor vibration mitigation. It can be estimated though by knowing the past states of the structure and control forces. The estimated disturbance is however delayed by at least one time step in reality $(\tau \geq T s)$. Starting from the closed loop transfer function (1) it follows the disturbance estimation $\hat{D}_{1}(s)$ :

$$
\hat{D}(s)=\hat{G}^{-1}(s) \cdot X(s) \mathrm{e}^{-s \tau}-F(s) \mathrm{e}^{-s \tau} \approx D(s)+\varepsilon(s)
$$

Let $G_{d}(s)$ be the desired closed loop behaviour of the structure. For this control strategy the auxiliary disturbance force $D_{2}$ is neglected. The closed loop behaviour is therefore (from equation $3, G_{i, k}$ are the components of $\bar{G}$ ):

$$
X_{1}(s)=G_{1,1}(s) \cdot\left(D_{1}(s)+F(s)\right)-G_{1,2} \cdot F(s)=G_{d}(s) \cdot D_{1}(s)
$$

The control law for the actuator force $F(s)$ is easily deduced from (8) and by inserting (7):

$$
F(s)=\left(\frac{G_{d}(s)-G_{1,1}}{G_{1,1}(s)-G_{1,2}(s)}\right) \cdot \hat{D}_{1}(s)=\frac{\mathrm{e}^{-s \tau}\left(G_{d}-G_{1,1}\right)}{G_{1,1}-G_{1,2}+\mathrm{e}^{-s \tau}\left(G_{d}-G_{1,1}\right)} \hat{G}^{-} 1(s) \cdot X(s)
$$

In the theoretic case of a perfect estimation $(\hat{G}=G)$ with time delay the closed loop behaviour is:

$$
\left[\begin{array}{l}
X_{1} \\
X_{2}
\end{array}\right]=G \cdot\left[\begin{array}{c}
D+F \\
-F
\end{array}\right]=\left[\begin{array}{c}
G_{d} \mathrm{e}^{-s \tau}+G_{1,1}\left(1-\mathrm{e}^{-s \tau}\right) \\
\left.\frac{1}{G_{1,1}-G_{1,2}}\left[G_{1,1} G_{2,1}-G_{1,2} G_{2,1}-\mathrm{e}^{-s \tau}\left(G_{1,1} G_{2,1}-G_{1,1} G_{2,2}-G_{2,1} G_{d}+G_{2,2} G_{d}\right)\right)\right]
\end{array}\right] D_{1}
$$

Equation (10) shows that in theory every desired closed loop behaviour $G_{d}(s)$ can be achieved for $\tau \rightarrow 0$. Stability and robustness of the close loop system must be analysed separately. When taking into account the time delay due to the estimation, the initial structure $G_{1,1}$ appears in the closed loop behaviour. For the simulations a close loop behaviour with a damping ratio of $20 \%$ and an estimation time delay of $1 \mathrm{~ms}$ is chosen.

\section{Results and discussion}

The control laws described in section 2 have been simulated using a linear model in MATLAB/SIMULINK. The frequency response plots are shown in figure 2 and 3.

\subsection{Tuned Mass Damper}

The simulated tuned mass damper provides good results for the case where only one resonance frequency is present. A reduction of the resonance peak of $-17 \mathrm{~dB}$ is achieved. The drawback of this type is that only one resonance peak can be mitigated, which is shown clearly on figure $2 b$. 


\subsection{Velocity feedback}

Velocity feedback is a very simple and easy to implement, but also efficient control law. In the case of only one resonance the controller gain of $4^{\prime} 500 \mathrm{~kg} / \mathrm{s}$ achieves an improvement of the peak resonance of $-17 \mathrm{~dB}$ (cf. figure 2a). The drawback is that the auxiliary system is strongly amplified in the low frequency domain. Tests with a controller gain of $5^{\prime} 000 \mathrm{~kg} / \mathrm{s}$ have led to an unstable system. Improvements can be achieved when an additional viscous damping of the auxiliary system is provided by the actuator. The increased damping has only little effect on the overall damping efficiency. The main advantage of the velocity feedback is the ability to mitigate even systems with multiple resonances. Figure $2 b$ shows that both peaks are equally reduced. Again the simple velocity feedback leads to high resonance amplitudes of the auxiliary system which can be improved by adding an artificial viscous damping force.

\subsection{Acceleration feedback}

The positive acceleration feedback proposed by Nishimura [2] provides very good results in the area of the structures resonance peak (cf. figure $3 \mathrm{a}$ ). A peak resonance improvement of $-31 \mathrm{~dB}$ is achieved compared to the undamped system. The drawback of this method is that high frequencies are amplified by about $3 \mathrm{~dB}$. On a real system this can lead to the amplification of higher order modes. This phenomena appears also in the case of the system with two resonance frequencies on figure $3 \mathrm{~b}$. Disturbances are strongly amplified in the frequency range above $5 \mathrm{~Hz}$.

\subsection{Disturbance Estimation}

For the estimation of the disturbance a very precise model of the structure is required. Also the estimation time delay is crucial for the damping efficiency. This theoretic approach shows (fig. 3a) that the desired transfer function can be nearly achieved. The small peak at the resonance frequency is due to the chosen estimation time delay of $1 \mathrm{~ms}$ in this simulation. By reducing the time delay this phenomena disappears. On the other hand this theoretic approach shows that the auxiliary mass is going to infinity for static disturbances. For a real application appropriate high-pass filtering needs to be considered. The same phenomena is visible for the case of a system with two resonance frequencies in figure $3 \mathrm{~b}$. It is clear that this control strategy works only for well-defined systems with little variation and nonlinearities.

\section{Discussion and Outlook}

The simulation results show that the TMD, velocity or acceleration feedback and the disturbance estimation work well for the mitigation of floor vibrations. Each of these strategies present advantages and drawbacks. For the vibration mitigation of floors with varying modal mass certainly, a disturbance estimation is difficult to establish. The TMD and acceleration feedback according to Nishimura are good solutions for structures with clearly separated resonance frequencies. The velocity feedback shows a good damping efficiency for both analysed systems. This strategy is able to mitigate even varying resonance frequencies. An artificial viscous damping of the auxiliary system reduces strongly the risk of instabilities. Some of these control strategies are currently analysed on a laboratory test bench. More detailed information on active vibration control is available in [5].

\section{References}

[1] Gutierrez S., Hojjat, A., Tuned Mass Dampers, Archives of Computational Methods in Engineering 20 (2013) 419-431

[2] I. Nishimura et al., Active tuned mass damper, Smart Mater Struct 1 (1992) 306-311

[3] F. Weber et al., Aktiv geregelte Schwingungstilger für weitgespannte Förderbandbrücken, Stahlbau 84 (2015) 246-247

[4] Hudson, E. et al., Fundamental Studies of AVC with Actuator Dynamics, Dynamics of Coupled Structures, Volume 4: Proceedings of the 34th IMAC, A Conference and Exposition on Structural Dynamics 2016 (2016) 147-154

[5] A. Preumont, Vibration Control of Active Structures, 3rd edition, Springer (2011), Berlin 


\begin{tabular}{cccc}
\hline System & Frequency [Hz] & Modal Mass [kg] & Damping ratio [\%] \\
\hline 1 & 3.0 & $5^{\prime} 000$ & 0.5 \\
\hline \multirow{2}{*}{2} & 3.0 & $5^{\prime} 000$ & 0.5 \\
& 4.0 & $3^{\prime} 000$ & 1.0 \\
\hline
\end{tabular}

Table 1: System simulation parameters

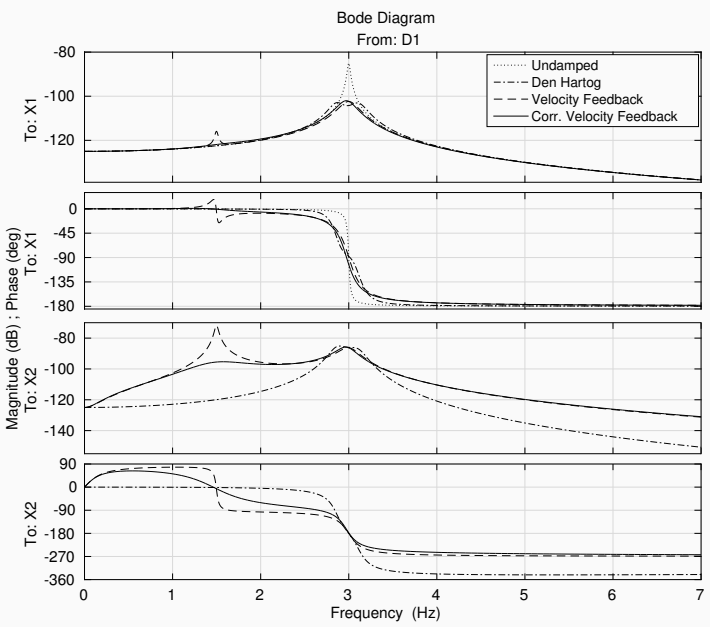

(a) 2nd order system

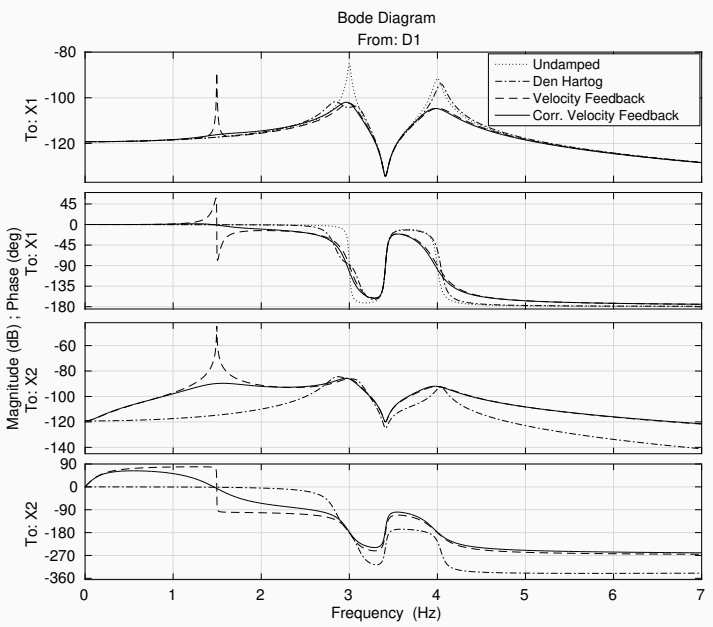

(b) 4th order system

Fig. 2: FRF of the undamped structure, the passive TMD according to Den Hartog, the uncorrected velocity feedback system and the corrected velocity feedback system. The upper graph shows the magnitude and phase of the position of the structure $X_{1}$ due to a harmonic disturbance $D_{1}$, whereas the lower graph is the magnitude and phase of the auxiliary system $X_{2}$ to the disturbance $D_{1}$

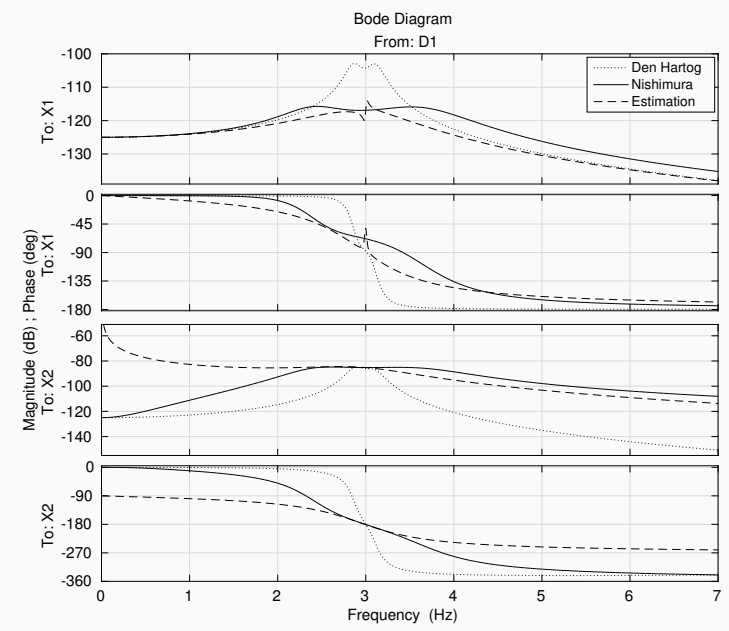

(a) 2nd order system

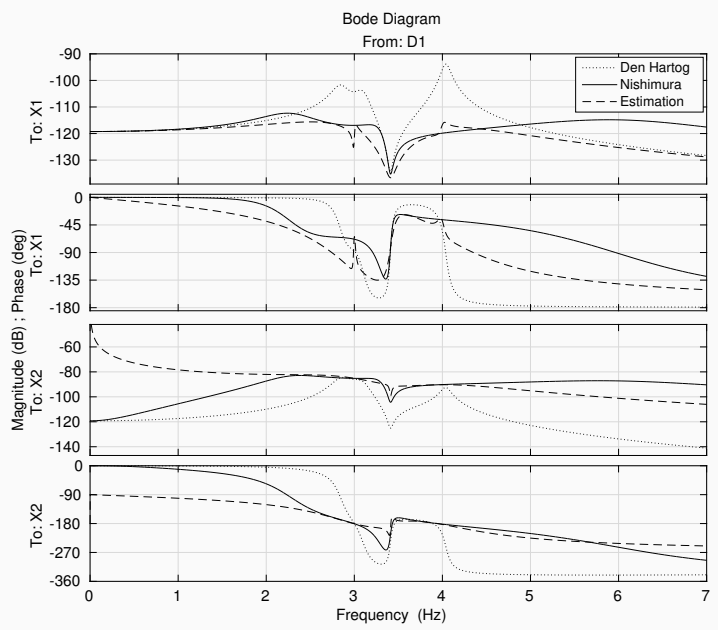

(b) 4 th order system

Fig. 3: FRF of the Den Hartog tuned TMD (as reference), the acceleration feedback system and the closed loop system using an estimator. The upper graph shows the magnitude and phase of the position of the structure $X_{1}$ due to a harmonic disturbance $D_{1}$, whereas the lower graph is the magnitude and phase of the auxiliary system $X_{2}$ to the disturbance $D_{1}$ 